\title{
Das Obersthofmarschallamt als Sondergericht des Kaiserhauses 1815-1918/19
}

\author{
The Lord High Steward's Office (Obersthofmarschallamt) \\ as Special Court of the Imperial Family 1815-1918/19
}

The Lord High Steward (Obersthofmarschall) was one of the four highest officers at the Habsburg court. Until the end of the 18 th century the Lord High Steward's office was a court for all members of the Imperial household. In 1815 it became a special court for members of the Imperial family. This article deals with the jurisdiction and the procedure of the Lord High Steward's office as the court of the Imperial family.

Keywords: Austria-Hungary - Imperial family - Imperial household - Obersthofmarschallamt

\section{Entwicklung und Zuständigkeit}

Der Obersthofmarschall war seit den Reformen Ferdinands I. eine von vier Obersten Hofchargen im Hofstaat der Habsburger. Zu seinen wichtigsten Aufgaben gehörte die Gerichtsbarkeit über die Personen des Hofstaats. ${ }^{1}$ Joseph II. hob diese Sondergerichtsbarkeit 1783 weitestgehend auf und unterstellte die Angehörigen des Hofstaats den allgemeinen Gerichten. Trotzdem blieb das Obersthofmarschallamt als Gerichtsbehörde bestehen; seine Zuständigkeit wurde aber auf Rechtsangelegenheiten des Personals des Reichshofrats beschränkt. ${ }^{2}$ Zwei Jahre später wurde das Obersthofmarschallamt auch für Rechtssachen von Botschaften und Gesandtschaften zuständig. ${ }^{3}$ Während die Zuständigkeit

\footnotetext{
${ }^{1}$ Zur Geschichte des Obersthofmarschallamts siehe: STROBL, Obersthofmarschallamt; ZOLGER, Hofstaat 104-117; WINKELHOFER, Alltag 63-65.

${ }^{2}$ Entschließung vom 5.8.1783, zitiert bei STROBL, Obersthofmarschallamt 113-114.

${ }^{3}$ JGS 481/1785.
}

für Rechtsangelegenheiten des Reichshofsrats mit dem Ende des Alten Reichs zwangsläufig wegfiel, blieb die Zuständigkeit des Obersthofmarschallamts für exterritoriale Personen bis zum Ende der Monarchie bestehen. Seine im 19. Jahrhundert wichtigste Kompetenz erhielt das Obersthofmarschallamt - nach einzelnen Vorstufen ${ }^{4}$ - schließlich 1815: In einem Hofdekret normierte Kaiser Franz II./I., dass das Amt für alle Zivilrechtsangelegenheiten von Mitgliedern des Kaiserhauses zuständig sein sollte. ${ }^{5}$ Dies wurde auch im Familienstatut des Kaiserhauses von $1839^{6}$ bestätigt. Die Oktroyierte Märzverfassung von 1849 bestätigte wieder „die

\footnotetext{
${ }^{4}$ Beispielsweise handelte das Obersthofmarschallamt bereits die Verlassenschaft nach Joseph II. ab (STROBL, Obersthofmarschallamt 115); zur Entwicklung bis 1815 siehe: ebd. 115-119.

5 JGS 1159/1815.

${ }^{6} \S 27$ Familienstatut;. Das Familienstatut befindet sich im HHStA, Familienurkunden Nr. 2362; eine (teilweise fehlerhafte) Transkription findet sich unter: http://www.heraldica.org/topics/royalty/hg1839.htm (abgerufen am 11. 3 2016).
} 
Bestimmungen der Hausgesetze [also des Familienstatuts] über den Gerichtsstand der Glieder des kaiserlichen Hauses. "7 Allerdings blieb das Familienstatut als Hausgesetz der Habsburger bis zum Ende der Monarchie geheim, weshalb seine Rechtsnatur unklar war. ${ }^{8}$

Neben der Zuständigkeit für Exterritoriale und Mitglieder des Kaiserhauses konnte der Gerichtsstand vor dem Obersthofmarschallamt auch als Privileg an einzelne Personen verliehen werden, die in der Praxis ausnahmslos dem Hochadel entstammten. ${ }^{9}$

Die Kompetenzen des Obersthofmarschallamts, wie sie sich seit Joseph II. entwickelt hatten, wurden in Art. V der JN 1852 bestätigt und zum ersten Mal an einer Stelle zusammenfassend geregelt:

„Das Obersthofmarschallamt wird in der Ausübung der Gerichtsbarkeit über die Mitglieder des kaiserlichen Hauses und über Personen, welchen die Exterritorialität zusteht, oder durch besondere Anordnungen eingeräumt wurde, erhalten. “ ${ }^{10}$

Auch die große Reform von 1895 änderte die Zuständigkeit des Obersthofmarschallamtes nicht. Art. III der JN 1895 war zwar anders formuliert als die Vorgängerbestimmung von 1852, der normative Gehalt war aber praktisch derselbe:

„Das Obersthofmarschallamt wird erhalten in der Ausübung der Gerichtsbarkeit:

1. über die Mitglieder des kaiserlichen Hauses;

2. über Personen, auf welche die Gerichtsbarkeit des Obersthofmarschallamtes ausgedehnt wur-

\footnotetext{
${ }^{7} \S 106$ Oktroyierte Märzverfassung (RGBl. 150/1849); das ist interessanterweise der einzige Verweis auf die Gerichtsbarkeit des Obersthofmarschallamts in einer Verfassung bzw. einem offiziellen Verfassungsentwurf.

${ }^{8}$ HAUKE, Kaiserliches und königliches Haus.

${ }^{9}$ Eine Aufzählung aller dieser Personen ist enthalten in: STROBL, Obersthofmarschallgericht 735.

${ }^{10}$ RGBl. 251/1852.
}

de oder in Hinkunft ausgedehnt wird;

3. über Personen, welchen die Exterritorialität zusteht, falls sie sich der Gerichtsbarkeit des Obersthofmarschallamtes unterwerfen; ${ }^{\prime \prime 11}$

Der Aufgabenbereich des Obersthofmarschallamts hatte sich somit vom 18. zum 19. Jahrhundert grundlegend gewandelt. Es war und blieb ein Sondergericht, aber bis zum 18. Jahrhundert war es ein Sondergericht für Angehörige des Hofes, danach wurde es zum Sondergericht für das Kaiserhaus. Freilich war das Obersthofmarschallamt nicht ausnahmslos für alle möglichen Gerichtsverfahren von Mitgliedern des Kaiserhauses zuständig. Im 19. Jahrhundert wurde es nämlich zu einem reinen Zivilgericht. ${ }^{12}$

Für die Zuständigkeit in allfälligen Strafverfahren gegen Mitglieder des Kaiserhauses gab es keine gesetzliche Regelung. ${ }^{13}$ Nur in $\S 33$ des Familienstatuts wurde für diesen Fall festgelegt: "Obwohl Wir endlich in der beruhigenden Überzeugung stehen, und es von Gott als eine Gnade des Himmels erflehen, daß der Fall nie eintreten möge, wo ein strafrechtliches Verfahren gegen ein Glied Unseres kaiserlichen Hauses einzutreten hätte, so wollen Wir doch Uns sowohl als Unsern Regierungsnachfolgern für einen solchen Fall ausdrücklich vorbehalten, nach den jedesmaligen Umständen jene Verfügungen zu treffen, welche die Handhabung des Rechtes, das Wohl des Staates, so wie die Ehre und das Beste Unseres Hauses erfordern werden." Die Entscheidung, ob, wie und wo ein Strafverfahren gegen ein Mitglied des Kaiserhauses durchgeführt werden sollte, behielt sich der Kaiser also für den Einzelfall vor. Tatsäch-

\footnotetext{
${ }^{11}$ RGBl. 110/1895.

${ }^{12}$ Die letzten polizeilichen Befugnisse in Bezug auf das Hofpersonal verlor das Obersthofmarschallamt 1849; RGBl. 269/1849; dazu: ZoLGER, Hofstaat 114.

${ }^{13}$ Strafverfahren gegen den Kaiser waren wegen durch dessen Unverantwortlichkeit ausgeschlossen; gegen andere Mitglieder des Kaiserhauses waren sie (theoretisch) möglich.
} 
lich dürfte es nie zu einem derartigen Verfahren gekommen sein.

Auch im Zivilrecht war das Obersthofmarschallamt nicht für alle Rechtsangelegenheiten des Kaiserhauses zuständig. Streitigkeiten zwischen Mitgliedern des Kaiserhauses untereinander sollten nämlich durch ein eigenes, ad hoc ausgewähltes Schiedsgericht entschieden werden. Diese Nicht-Zuständigkeit des Obersthofmarschallamts ergab sich interessanterweise nicht aus einem staatlichen Gesetz, sondern lediglich aus dem Familienstatut. ${ }^{14}$

Eine weitere Ausnahme von der Zuständigkeit des Obersthofmarschallamts für Rechtsstreitigkeiten des Kaiserhauses ergab sich aus dem generellen Zuständigkeitssystem des österreichischen Zivilverfahrens. Das Obersthofmarschallamt war ein besonderer Gerichtsstand für das Kaiserhaus. Wie andere Gerichtsstände auch kam dieser Gerichtsstand nur dann zur Anwendung, wenn ein Mitglied des Kaiserhauses geklagt wurde. ${ }^{15}$ Wenn hingegen ein Erzherzog oder eine Erzherzogin selbst klagte, richtete sich

${ }^{14} \S 28$ „Unter die von der Gerichtbarkeit des Oberhofmarschallamtes ausgenommenen Fälle gehören insbesondere jene, in welchen unter den Mitgliedern des Erzhauses selbst privatrechtlichen Streitigkeiten entstehen sollten. In solchen Fällen haben die streitenden Theile Schiedsrichter $\mathrm{zu}$ wählen und dem Oberhaupte der Familie unter Beilegung des Kompromisses zur Bestättigung anzuzeigen. Sollten sie sich über die Wahl der Schiedsrichter nicht vereinigen können, so bleibt dem Oberhaupte der Familie deren Benennung vorbehalten. Die Schiedsrichter haben die Verhandlung nach den allgemein bestehenden Gesetzen $\mathrm{zu}$ leiten und $\mathrm{zu}$ entscheiden. Von dieser Entscheidung hat eine Berufung an das Oberhaupt der Familie nur dann Statt, wenn sich die streitenden Theile diese Berufung in dem Kompromiße ausdrücklich vorbehalten, und auch in diesem Punkte die Bestättigung des Kompromisses erlangt haben."

${ }^{15}$ So auch $\S 32$, 1. Satz Familienstatut: „Die nicht ausgenommen Rechtsfälle, woran die Mitglieder Unseres Erzhauses als Beklagte Theil nehmen, sind bei dem Obersthofmarschallamte $\mathrm{zu}$ verhandeln und $\mathrm{zu}$ entscheiden." die Zuständigkeit grundsätzlich nach dem Gerichtsstand des Beklagten. Eine Besonderheit des Gerichtsstandes vor dem Obersthofmarschallamt bestand weiters darin, dass er unprorogabel war, also durch Parteienvereinbarung nicht abgeändert werden konnte. ${ }^{16}$

Schließlich war das Obersthofmarschallamt auch dann nicht zuständig, wenn Mitglieder des Kaiserhauses wegen Liegenschaften geklagt wurden. In solchen Fällen ging der Realgerichtsstand, der Gerichtsstand der gelegenen Sache jenem vor dem Obersthofmarschallamt vor. ${ }^{17}$

In der Praxis waren Zivilprozesse keineswegs das wichtigste Tätigkeitsgebiet des Obersthofmarschallamts. ${ }^{18}$ Der weitaus größte Teil der Verfahren, die das Amt abwickelte, waren AuBerstreitangelegenheiten, wie insbesondere Verlassenschaftsabhandlungen. ${ }^{19}$ Außerdem war das Obersthofmarschallamt für Exekutionen gegen Mitglieder des Kaiserhauses zuständig. ${ }^{20}$

\section{Verfahren}

Für das Verfahren vor dem Obersthofmarschallamt legte Joseph II. 1785 fest, dass das Amt „in jenen Angelegenheiten, die nicht vom Reichshofrathe dahin kommen, und nach den Reichsgesetzen zu behandeln sind, sich der eingeführ-

\footnotetext{
${ }^{16} \S 32$, 2. Satz Familienstatut: „Diesem Gerichtsstande kann durch Vertrag nicht entsagt werden."

${ }^{17} \S 32$, 3. und 4. Satz Familienstatut; „Die Jurisdiction des Obersthofmarschallamtes erstreckt sich jedoch nicht auf dingliche Klagen, die wegen unbeweglicher Güter gegen Mitglieder der durchlauchtigen Familie angebracht werden. Für diese bleibt es bei der allgemeinen Vorschrift über den Gerichtsstand der gelegenen Sache."

18 BACHRACH, Regierungsvorlage.

${ }^{19}$ Die Akten des Obersthofmarschallamts befinden sich im ÖStA, HHStA, Hofarchive; ein prominentes Beispiel für ein vor dem Amt abgewickeltes Verlassenschaftsverfahren ist jenes nach Kaiser Franz Joseph; dazu SCHMETTERER, Letztwillige Verfügungen. ${ }^{20} \S 31$ EO 1896 (RGBl. 79/1896).
} 
ten erbländischen Justiz-Ordnung zu fügen“ hatte. ${ }^{21}$ Die damals in den Erblanden eingeführte Verfahrensordnung war die AGO von 1781,22 die daher auch vom Obersthofmarschhallamt angewendet wurde.

In seiner Besetzung unterschied sich das Obersthofmarschallamt aber von anderen Gerichten. In streitigen Verfahren entschied es wie die Appellationsgerichte nach der $\mathrm{AGO}^{23}$ als Fünfersenat; am Obersthofmarschallamt war aber nur der Kanzleidirektor als Vorsitzender hauptamtlich tätig. Die vier Beisitzer wurden von anderen Wiener Gerichten beigezogen. ${ }^{24}$ Der Hintergrund für diese Konstruktion war, dass Joseph II. dem Obersthofmarschallamt praktisch alle Zuständigkeiten genommen hatte. Für die kleine Restzuständigkeit in bestimmten Angelegenheiten des Reichshofrats war hauptamtliches richterliches Personal nicht erforderlich; daher wurden Richter anderer Gerichte eingesetzt.

Diese Entscheidungsform durch beigezogene Richter wurde auch beibehalten, als die Kompetenzen des Obersthofmarschallamts wieder erweitert wurden und das Amt auch eigenes hauptamtliches juristisches Personal erhielt. Allerdings wurde diese Entscheidungsfindung nur in Zivilprozessen angewendet. In Außerstreitangelegenheiten entschied hingegen der Kanzleidirektor alleine. Seine Entscheidungen wurden von den drei hauptamtlichen Kon-

\footnotetext{
${ }^{21}$ JGS 481/1785.

${ }^{22}$ JGS 13/1781; dazu: LOSCHELDER, Allgemeine Gerichtsordnung.

${ }^{23}$ WALDSTÄTTEN, Staatliche Gerichte 51.

${ }^{24}$ Bis in die Mitte des 19. Jhds. waren die Beisitzer Richter des niederösterreichischen Appellationsgerichts (bei adeligen Beklagten) oder des Wiener Magistrats (bei nichtadeligen Beklagten); JGS 481/1785. Danach wurden Richter der Wiener Gerichtshöfe erster Instanz (also des Landesgerichts und/oder des Handelsgerichts) als Beisitzer am Obersthofmarschallamt herangezogen; STROBL, Obersthofmarschallamt 124.
}

zeptsbeamten vorbereitet, die das Amt im Lauf des 19. Jahrhunderts erhalten hatte, und mussten vom Obersthofmarschall genehmigt werden. ${ }^{25}$

Als das Obersthofmarschallamt 1815 für die Mitglieder des Kaiserhauses zuständig wurde, ergingen keine besonderen Regelungen für das Verfahren in derartigen Fällen. Somit war die AGO auch in Verfahren anwendbar, an denen Mitglieder des Kaiserhauses beteiligt waren. Im Familienstatut wurden dann allerdings zwei Besonderheit für derartige Verfahren normiert: Die Mitglieder des Kaiserhauses waren erstens nicht zur Eidesleistung verpflichtet; bei Ihnen reichte vielmehr eine schriftliche Erklärung an Eides statt. ${ }^{26}$ Zweitens sollten Verfahren mit Beteiligung von Erzherzögen oder Erzherzoginnen nach Möglichkeit durch einen Vergleich beendet werden. Das wurde im Familienstatut besonders betont. ${ }^{27}$ Daraus lässt sich ableiten, dass eine vergleichsweise Einigung in Verfahren mit Mitgliedern des Kaiserhauses eine noch größere Priorität haben sollte als in anderen Verfahren. Dies wurde in der Verordnung über das Obersthofmarschallamt von 1916 bestätigt und insofern präzisiert, als diese Verordnung einen Vergleichsversuch für die Verfahren vor dem Amt zwingend vorschrieb. ${ }^{28}$

Seit 1785 war das Obersthofmarschallamt in den allgemeinen Instanzenzug eingegliedert. Über Urteile des Amtes sollte in zweiter Instanz das niederösterreichische Appellationsgericht entscheiden und in dritter und letzter die oberste Justizstelle. ${ }^{29}$ Dieser Instanzenzug wurde im Familienstatut ausdrücklich auch auf Verfahren gegen Mitglieder des Kaiserhauses ausge-

\footnotetext{
25 STROBL, Obersthofmarschallamt 123-124.

${ }^{26} \S 31$ Familienstatut.

$27 \S 32$ letzter Satz Familienstatut.

${ }^{28} \S 2$ Z. 1 RGBl. 223/1916.

${ }^{29}$ Hofdekrete vom 14.10.1785 (JGS 481/1785) und vom 30. 1. 1795 (JGS 216/1795).
} 
dehnt. ${ }^{30}$ Durch die Neuordnung der Gerichtsorganisation trat nach 1848 das Oberlandesgericht Wien an die Stelle des niederösterreichischen Appellationsgerichts und der Oberste Gerichtsund Cassationshof an jene der Obersten Justizstelle. ${ }^{31}$

Das streitige Verfahren vor dem Obersthofmarschallamt blieb fast während des gesamten 19. Jahrhunderts unverändert - und sogar darüber hinaus. Bei der großen Reform des Zivilprozessrechts in den 1890er-Jahren stellte sich nämlich die Frage, ob diese Reform auch das Obersthofmarschallamt miteinbeziehen sollte. Konkret ging es darum, was die Formulierung von der „eingeführten erbländischen JustizOrdnung" im Dekret von 1785 genau bedeuten sollte. War damit die 1785 geltende AGO gemeint und nur diese, oder sollte das Obersthofmarschallamt vielmehr die jeweilige allgemeine Verfahrensordnung anwenden? Die Frage war also - modern formuliert -, ob es sich bei jenem knappen Hinweis um eine statische Verweisung (nur auf die AGO) oder um eine dynamische Verweisung (auf jedes gerade geltende Verfahrensrecht) handeln sollte. ${ }^{32}$

Bei der Erlassung der neuen Justizgesetze 1895 löste der Gesetzgeber diese Frage autoritativ und legte in Art. III, letzter Absatz EGJN fest: „Die im Hofdecret vom 14. October 1785, J.G.S. Nr. 481, in Ansehung des Instanzenzuges getroffenen Bestimmungen, sowie die Vorschriften für das Verfahren in den zur Gerichtsbarkeit des Obersthofmarschallamtes gehörigen Rechtssachen bleiben unberührt." Damit wurde festgelegt, dass das Obersthofmarschallamt weiterhin die AGO anwenden sollte, obwohl der Wortlaut dieser Bestimmungen des EGJN streng genommen auch nicht klärte, ob die Verweisung von

\footnotetext{
${ }^{30} \S 27$ Familienstatut.

31 OGRIS, Rechtsentwicklung 550-551; WALDSTÄTTEN, Staatliche Gerichte 84-108.

32 Siehe dazu: SCHRUTKA, Regierungsvorlage; BACHRACH, Regierungsvorlage.
}

1785 eine statische oder eine dynamische war. Im Kontext war aber klar, dass das Obersthofmarschallamt auch nach der Reform weiter die AGO und nicht die neue ZPO anwenden sollte sonst wäre die Bestimmung in Art. III, letzter Absatz EGJN gar nicht erforderlich gewesen.

Ein konkreter Grund für diese Entscheidung war der Grundsatz der Öffentlichkeit, der mit der ZPO allgemein in den Zivilprozess eingeführt wurde. Die damit verbundene potentielle publicity war für Verfahren gegen Mitglieder des Kaisershauses nicht erwünscht. ${ }^{33}$ Dazu kam noch eine allgemeine Tendenz zur besonderen Bewahrung traditioneller Formen im Zusammenhang mit dem kaiserlichen Hof.

Die Beibehaltung der AGO für das Obersthofmarschallamt wurde bald kritisiert. Als Eduard Strobl Ritter von Albeg 1908 eine umfangreiche Geschichte dieses Amts veröffentlichte, beklagte er am Schluss des Kapitels über die damals geltende Rechtslage, dass dem Amt "die so große Wohltat der Zivilprozeßreform nicht beschieden" worden sei, und schloss dann mit dem eindringlichen Appell: „möge diesem ältesten Gericht Österreichs eine seiner hohen Aufgabe würdige Neugestaltung beschieden sein. “"

Tatsächlich brachte die Beibehaltung einer antiquierten Verfahrensordnung für ein einziges Gericht eine Reihe praktischer Probleme mit sich. Man konnte zwar davon ausgehen, dass das ständige Personal des Obersthofmarschallamtes weiterhin mit dem Verfahren nach der AGO vertraut bleiben würde. Für jene vier Richter aber, die in Zivilprozessen vor dem Amt als Beisitzer fungierten, bedeutete die Beibehaltung der AGO, dass sie in den seltenen Prozessen vor dem Obersthofmarschallamt nach einem grundlegend anderen Verfahren entscheiden mussten als in ihrem beruflichen Alltag. Vor allem aber musste man davon ausgehen, dass es mit der

\footnotetext{
33 WINKELHOFER, Alltag 65.

34 STROBL, Obersthofmarschallamt 125.
} 
Zeit kaum noch Anwälte geben würde, die mit der AGO vertraut waren und damit ein Verfahren vor dem Obersthofmarschallamt führen konnten. Genauso hätten die Richter am Oberlandesgericht oder am Obersten Gerichts- und Cassationshof bei Rechtsmitteln gegen Entscheidungen des Obersthofmarschallamtes nach einem Verfahren entscheiden müssen, das sie sonst überhaupt nicht mehr anwendeten. ${ }^{35}$

1909 wurde in Budapest ein eigenes ungarisches Hofmarschallgericht eingerichtet (siehe dazu den folgenden Abschnitt). ${ }^{36}$ Dieses neugeschaffene ungarische Hofgericht wendete von Anfang an das zeitgenössische ungarische Zivilverfahrensrecht an, und daher wurde auch das vor dem Obersthofmarschallamt in Wien anzuwendende Verfahren überdacht: 1910 brachte die Regierung eine Vorlage für ein „Gesetz über die Ausübung der Gerichtsbarkeit durch das Obersthofmarschallamt" im Herrenhaus des Reichsrats ein..$^{37} 1910$ wurde diese Vorlage vom Herrenhaus nicht beschlossen und so wurde im Jahr darauf noch einmal eine weitgehend gleichlautende Regierungsvorlage im Herrenhaus eingebracht..$^{38}$ Die neue Regierungsvorlage wurde im Herrenhaus 1912 unverändert angenommen, ${ }^{39}$ blieb im Abgeordnetenhaus aber bis zur Vertagung des Reichsrats in Frühjahr 1914 unerledigt. Erst 1916 erließ Kaiser Franz Joseph eine Verordnung gemäß § 14 StGG-RV, mit der die

\footnotetext{
${ }^{35} \mathrm{RV}$, StProt HH, XXI. Session, Blg. 18.

${ }^{36}$ GA XVI:1909.

${ }^{37} \mathrm{RV}$, StProt HH, XX. Session, Blg. 77; dazu: BACH$\mathrm{RACH}$, Regierungsvorlage; SCHRUTKA, Regierungsvorlage.

${ }^{38} \mathrm{RV}$, StProt HH, XXI. Session, Blg. 18. In der Vorlage von 1911 wurde $\S 5$ des Gesetzesvorschlages von 1910 nicht übernommen, der ausdrücklich klargestellt hatte, dass der Gerichtsstand der gelegenen Sache jenem vor dem Obersthofmarschallamt vorging. Dafür gab es in der Vorlage von 1911 einen zusätzlichen Absatz in $\S 4$, in dem festgehalten wurde, dass bestimmte Besonderheiten des Verfahrens auch in der zweiten und dritten Instanz anzuwenden sein sollten. ${ }^{39}$ StProt HH, XXI. Session, 216-217.
}

ZPO mit leichten Modifikationen auch für das Obersthofmarschallamt eingeführt wurde. ${ }^{40}$ Diese Verordnung entsprach inhaltlich der Regierungsvorlage von 1911.41 Nach der Wiedereinberufung des Reichsrats 1917 wurde derselbe Text nochmals als Regierungsvorlage im Reichsrat eingebracht, ${ }^{42}$ aber bis zum Ende der Monarchie nicht mehr beschlossen. Somit konnte das Obersthofmarschallamt die ZPO nur in den letzten Jahren seines Bestehens anwenden - und zwar bis zum Schluss aufgrund einer kaiserlichen Notverordnung.

Diese kaiserliche Notverordnung passte das Verfahren vor dem Obersthofmarschallamt an jenes vor anderen österreichischen Gerichten an, ließ aber einige Besonderheiten bestehen. Das galt nicht zuletzt für die Gerichtsbesetzung. $\mathrm{Ab}$ 1916 entschied das Obersthofmarschallamt entweder als Dreirichtersenat oder durch einen Einzelrichter. Dies entsprach der Besetzung der anderen erstinstanzlichen Gerichte in Zivilsachen. Allerdings war die Aufteilung von Einzelrichter- und Senatszuständigkeit am Obersthofmarschallamt ein bisschen anders als in der sonstigen Gerichtsbarkeit: Die Bezirksgerichte entschieden über Streitwerte bis zu 500 Gulden $(=1.000 \text { Kronen })^{43}$ immer durch Einzelrichter, die Gerichtshöfe bis 1914 immer durch Dreiersenate; mit der in diesem Jahr ergangenen ersten Gerichtsentlastungsnovelle wurde die Entscheidung durch Einzelrichter auch an den Gerichtshöfen eingeführt - und zwar für Verfahren mit Streitwerten bis 2.500 Kronen. ${ }^{44}$ In streitigen Verfahren vor dem Obersthofmarschallamt war die Einzelrichterzuständigkeit hingegen viel

\footnotetext{
${ }^{40}$ RGBl. 223/1916.

${ }^{41}$ Lediglich in $\S 3$ wurde nach der Wortfolge "Die übrigen [...] bürgerlichen Rechtssachen“ der erklärende Klammerausdruck „(außerstreitige Angelegenheiten, Exekutionen u. a.)“ eingefügt.

${ }^{42} \mathrm{RV}$, StProt AH, XXII. Session, Blg. 143.

${ }^{43} \S 49$ Z. 1 JN.

${ }^{44} \S 7$ a JN (eingefügt durch die 1. Gerichtentlastungsnovelle, RGBl. 118/1914).
} 
eingeschränkter. Hier entschied ein Einzelrichter nur in Bagatellsachen, also in Verfahren mit einem Streitwert bis 50 Gulden (= 100 Kronen); ${ }^{45}$ in allen anderen streitigen Verfahren aber ein Dreirichter-Senat. ${ }^{46}$ Diese besonders weitgehende Senatszuständigkeit macht deutlich, dass den Verfahren vor dem Obersthofmarschallamt eine besondere Sensibilität und Bedeutung zugemessen wurde.

Auch nach der Einführung der ZPO auch für das Obersthofmarschallamt blieb es dabei, dass nicht alle dort tätigen Richter zum ständigen Personalstand des Amts gehörten. Nur der Vorsitzende (zuvor auch als Kanzleidirektor bezeichnet) und sein Stellvertreter wurden auf Lebensdauer an das Obersthofmarschallamt ernannt. ${ }^{47}$ Die beiden anderen Senatsmitglieder (und deren zwei Stellvertreter) waren weiterhin Richter, die an den Wiener Gerichtshöfen ernannt waren und dem Obersthofmarschallamt nur dienstzugeteilt wurden. Der Grund dafür lag vermutlich darin, dass am Obersthofmarschallamt nicht so viele Prozesse anfielen, dass drei hauptamtliche Richter dadurch ausgelastet gewesen wären. Die streitigen Verfahren bildeten nämlich nur einen vergleichsweise geringen Teil der praktischen Tätigkeit des Obersthofmarschallamts, und in allen anderen Angelegenheiten (Exekutionen, Außerstreitangelegenheiten) entschied das Amt durch Einzelrichter. ${ }^{48}$ Im Verfahrensablauf selbst sah die Verordnung von 1916 drei Besonderheiten vor: Das Obersthofmarschallamt musste vor der mündlichen Streitverhandlung einen Vergleichsversuch durchführen; die Öffentlichkeit konnte schon auf Antrag nur einer Partei ausgeschlossen werden, und Mitglieder des Kaiserhauses konnten

\footnotetext{
${ }^{45} \S 448 \mathrm{ZPO}$.

${ }^{46} \S 2$ Z. 4 RGBl. 223/1916.

${ }^{47} \S 1$ 1. Absatz RGB1. 223/1916.

${ }^{48} \S 3$ RGBl. 223/1916.
}

als Verfahrensparteien nicht zum persönlichen Erscheinen vorgeladen werden. ${ }^{49}$

Da die Öffentlichkeit schon auf Antrag einer Partei ausgeschlossen werden konnte, war sichergestellt, dass es nicht $\mathrm{zu}$ einem öffentliches Prozess gegen ein Mitglied des Kaiserhauses kommen konnte, wenn das Kaiserhaus die Öffentlichkeit vermeiden wollte. Damit war das vielleicht größte Hindernis für die Einführung der ZPO auch vor dem Obersthofmarschallamt ausgeräumt. Interessanterweise wurde diese Möglichkeit, die Öffentlichkeit auszuschließen, symmetrisch formuliert, so dass auch die nicht zum Kaiserhaus gehörende klagende Partei dies beantragen konnte. Im Gegensatz dazu war die Sonderbestimmung über die Vorladung von Parteien asymmetrisch formuliert. Nur Mitglieder des Kaiserhauses konnten nicht persönlich vorgeladen werden, andere Parteien hingegen schon. ${ }^{50}$

Es ist interessant, dass dem streitigen Verfahren vor dem Obersthofmarschallamt so viel Aufmerksamkeit gewidmet wurde, obwohl es in der praktischen Tätigkeit des Amtes nur eine untergeordnete Rolle spielte. Über das außerstreitige Verfahren, in dem das Obersthofmarschallamt viel mehr Fälle bearbeitete, gab es keine vergleichbaren Diskussionen. Bei der Einführung

\footnotetext{
49 § 2 RGBl. 223/1916.

${ }^{50} \mathrm{Im}$ Hinblick auf $\S 380$ Abs. 3 ZPO erscheint diese Bestimmung zunächst überflüssig, weil § 380 Abs. 3 ZPO normiert, dass keine Zwangsmaßnahmen angewendet werden dürfen, um Parteien zum Erscheinen oder zur Aussage zu zwingen. Der Sinn der Sonderbestimmung für Mitglieder des Kaiserhauses ergab sich wohl aus § 381 ZPO, der festlegt, dass das Nichterscheinen bzw. die Aussageverweigerung geladener Parteien vom Gericht im Sinne der freien Beweiswürdigung zu beurteilen ist. In aller Regel sind die Schlüsse, die ein Gericht aus dem Nichterscheinen einer Partei zieht, für diese Partei nicht günstig. Durch § 2 Z. 3 RGBl. 223/1916 wurde offenbar sichergestellt, dass das Obersthofmarschallamt aus dem Nicht-Erscheinen von Mitgliedern des Kaiserhauses keine negativen Schlüsse ziehen konnte.
} 
des Außerstreitgesetzes 1854 wurde keine Ausnahme für das Obersthofmarschallamt gemacht. ${ }^{51}$ Das mag damit zusammen hängen, dass das Außerstreitverfahren zuvor überhaupt nicht kodifiziert war, ${ }^{52}$ während es für das streitige Verfahren mit der AGO schon eine Kodifikation gegeben hatte, als die ZPO eingeführt wurde. Obwohl die Vorschriften von 1785 denkbar knapp waren, erscheint es immerhin vertretbar, dass mit der „eingeführten erbländischen Justiz-Ordnung" speziell die AGO gemeint war und nicht die Gesamtheit aller Einzelvorschriften zum gerichtlichen Verfahren sei es in streitigen, sei es in außerstreitigen Angelegenheiten. Das AußstrG 1854 wurde jedenfalls vom Obersthofmarschallamt angewendet; unter anderem im Verlassenschaftsverfahren nach Kaiser Franz Joseph, das erst im Herbst 1918 - gut einen Monat vor dem Ende der Monarchie - abgeschlossen werden konnte. ${ }^{53}$

\section{Das Obersthofmarschallamt in der Doppelmonarchie}

Nach dem Ausgleich von 1867 gab es in der Doppelmonarchie nur mehr wenige gemeinsame Angelegenheiten beider Reichshälften, und die ungarische Seite legte großen Wert darauf, dass dies so blieb und die Eigenständigkeit der Reichshälften möglichst stark betont wurde. Die staatsrechtliche Diskussion um Gemeinsamkeit und Eigenständigkeit machte auch vor der Person des Monarchen und seinem Hofstaat nicht halt. Radikale ungarische Politiker vertraten beispielsweise die Meinung, es gäbe gar keinen gemeinsamen Monarchen; vielmehr seien Kaiser von Österreich und König von Ungarn strikt zu trennende Funktionen, die nur zufällig von derselben Person ausgeübt würden. Die Konse-

\footnotetext{
${ }^{51}$ RGBl. 208/1854.

52 Dazu: OGRIS, Rechtsentwicklung 581-582.

53 SCHMETTERER, Letztwillige Verfügungen 337.
}

quenz aus dieser Auffassung wäre gewesen, dass es auch zwei völlig getrennte Hofstaaten gegeben hätte. Das konnte die ungarische Seite bis zum Ende der Monarchie nicht durchsetzen. ${ }^{54}$ Bis 1918 blieb es etwa bei den vier traditionellen Obersten Hofchargen, diese Funktionen wurden nicht auf jeweils einen österreichischen und einen ungarischen Obersthofmeister, Oberstkämmerer, Obersthofmarschall und Oberststallmeister aufgeteilt. In anderen Bereichen nahm Kaiser Franz Joseph auf die ungarischen Befindlichkeiten in den letzten Jahrzehnten seiner Regierung durchaus Rücksicht - wobei das nicht immer einen Nachteil für ihn bedeuten musste. So hatte der Monarch zum Beispiel sowohl einen österreichischen als auch einen ungarischen Hofzug. ${ }^{55}$

Die Gerichtsbarkeit war an sich jedenfalls eine autonome Angelegenheit der beiden Reichshälften. Nur in zwei Bereichen gab es ausnahmsweise gemeinsame Gerichte: Der eine waren die Militärgerichte der gemeinsamen (k.u.k.) Armee, der andere war das Obersthofmarschallamt. Um die Jahrhundertwende wurden auch in diesen Bereichen die ungarischen Unabhängigkeitsforderungen deutlich stärker. Eine Teilung der k.u.k. Armee konnte - wenn auch mit Zugeständnissen - vermieden werden, und so gab es bis zum Ende der Monarchie auch gemeinsame Militärgerichte. ${ }^{56}$ Im Bereich des Obersthofmarschallamts wurde als erstes Zugeständnis 1895 die Funktion eines Hofmarschalls in Ungarn geschaffen, der den Obersthofmarschall in Ungarn vertreten sollte, falls dieser nicht dort war. ${ }^{57}$ Ab 1901 wurde am Obersthofmarschallamt ein ständiger ungarischer Referent für Angelegenheiten mit einem Bezug zu Ungarn ein-

\footnotetext{
${ }^{54}$ Dazu Zolger, Hofstaat 310-382, insbesondere 310320 mit einem Überblick über die Diskussion.

${ }^{55}$ AICHELbURG, Franz Ferdinand 918-929.

${ }^{56}$ SCHMID, Heeresrecht 503-510.

${ }^{57}$ BERNATZIK, Verfassungsgesetze Nr. 25.
} 
geführt.58 1909 wurde schließlich in Budapest ein eigenes ungarisches Hofmarschallgericht errichtet, das ebenso wie das Obersthofmarschallamt in Wien dem Obersthofmarschall unterstellt war. ${ }^{59}$

Der grundsätzliche Wirkungskreis des neuen ungarischen Hofmarschallgerichts (Mitglieder des Herrscherhauses, Personen mit dem Privileg des Gerichtsstandes vor dem Obersthofmarschallamt, exterritoriale Personen) war mit jenem des Obersthofmarschallamts ident; ${ }^{60}$ das ungarische Gericht sollte dann zuständig sein, wenn es einen konkreten Bezug zu Ungarn gab. Bei Verlassenschaften war das dann der Fall, wenn es unbewegliches Nachlassvermögen in Ungarn gab oder die verstorbene Person ihren letzten ordentlichen Wohnsitz in Ungarn gehabt hatte. ${ }^{61}$ In anderen Fällen war das ungarische Obersthofmarschallgericht dann zuständig, wenn auch ohne besondere Hofmarschallgerichtsbarkeit ein ungarisches Gericht zuständig gewesen wäre. ${ }^{62}$

Diese Kriterien für die Zuständigkeitsabgrenzung zwischen Obersthofmarschallamt und ungarischem Hofmarschallgericht sind an sich sachlich und nachvollziehbar. Trotzdem wird bei genauerer Betrachtung deutlich, dass es kein organisatorisches Bedürfnis nach einer Aufteilung der Hofmarschallgerichtsbarkeit zwischen den beiden Reichshälften gab. Die wichtigste Zuständigkeit des Obersthofmarschallamts war jene für das Herrscherhaus und hier spielten Außerstreitsachen wie zum Beispiel Verlassenschaftsabhandlungen quantitativ die größte Rolle. Die Mitglieder des Herrscherhauses waren aber Angehörige beider Reichshälften ${ }^{63}$ und

\footnotetext{
${ }^{58}$ STROBL, Obersthofmarschallamt 124.

${ }^{59}$ GA XVI:1909.

${ }^{60}$ GA XVI:1909 § 3.

${ }^{61}$ GA XVI:1909 § 4 Z. 3.

${ }^{62}$ GA XVI:1909 § 4 Z. 4.

${ }^{63}$ Zur Frage ob die Angehörigen des Kaiserhauses Staatsbürger oder (sonstige) Staatsangehörige waren: SCHMETTERER, Mitglieder des Kaiserhauses 16-17;
}

viele hatten auch Wohnsitze in beiden Reichshälften, sodass die vermeintlich klaren Abgrenzungskriterien gerade in diesen zentralen Fällen eben nicht zu einem klaren Ergebnis führten. Das wird nicht zuletzt am Beispiel Kaiser Franz Josephs deutlich (wobei der Kaiser als Oberhaupt des Herrscherhauses und der damit verbundenen Souveränität eine gewisse Sonderstellung hatte): Franz Joseph hatte einen ordentlichen Wohnsitz in Ungarn und er hatte Liegenschaftsvermögen in Ungarn (zum Beispiel Schloß Gödöllö). Nach dem Wortlaut des $§ 4$ Z. 3 des GA XVI:1909 wäre daher das gesamte Verlassenschaftsverfahren nach dem Monarchen vor dem ungarischen Hofmarschallgericht in Budapest abzuführen gewesen. Das wäre allerdings wenig sachgerecht gewesen; immerhin hatte der Kaiser seit dem Beginn des Ersten Weltkriegs Wien nicht mehr verlassen und war auch in Schönbrunn gestorben. Außerdem hatte er in der österreichischen Reichshälfte wesentlich mehr Vermögen (bewegliches und unbewegliches) als in Ungarn. Schließlicht hatte der Kaiser selbst in seinem Testament vom 6. Februar 1901 angeordnet, dass seine Verlassenschaft vom Obersthofmarschallamt abgehandelt werden sollte. ${ }^{64}$ Allerdings gab es noch kein ungarisches Hofmarschallamt, als der Kaiser dieses Testament errichtete. Tatsächlich wurde das gesamte Verlassenschaftsverfahren nach Franz Joseph dann nach einigen Diskussionen vom Obersthofmarschallamt in Wien durchgeführt - nur die Inventarisierung der Nachlassteile in Budapest und Gödöllö wurde vom Hofmarschallgericht in Budapest durchgeführt. 65

An diesem prominenten Beispiel wird deutlich, dass die Abspaltung des ungarischen Hofmarschallgerichts vom Obersthofmarschallamt we-

ReIter-ZatloukAL, Staatsbürgerschaft als Politikum 102-104.

${ }^{64}$ SCHMETTERER, Letztwillige Verfügungen 327.

65 SCHMETTERER, Letztwillige Verfügungen 330. 
der klare Zuständigkeiten noch eine Vereinfachung des Verfahrens brachte. Das ungarische Hofmarschallgericht wurde nur errichtet, um den ungarischen Wünschen nach Parität und möglichst starker Unabhängigkeit der beiden Reichshälften zu entsprechen - und war damit symptomatisch für die staatsrechtlichpolitischen Probleme der Habsburgermonarchie in deren letzten Lebensjahren.

\section{Die Einbindung des Obersthofmarschallamts in die staatliche Gerichtsbarkeit}

Das Obersthofmarschallamt war ursprünglich eine besondere Form eines Patrimonialgerichts, das für das Personal am Hof der Habsburger zuständig war. Auch als die Patrimonialgerichtsbarkeit in der Habsburgermonarchie in Folge der Grundentlastung aufgehoben wurde, blieb das Obersthofmarschallamt als Gericht bestehen, wobei sich seine Zuständigkeit vom Ende des 18. zum Beginn des 19. Jahrhunderts bekanntlich so verschoben hatte, dass es nicht mehr für das Hofpersonal, sondern für die Mitglieder des Kaiserhauses zuständig war. Auch mit dieser neuen Kompetenz blieb das Obersthofmarschallamt ein Sonderfall in der Gerichtsorganisation der Donaumonarchie und bildete bis zum Ende der Monarchie einen letzten Rest der Patrimonialgerichtsbarkeit.

Das Obersthofmarschallamt hatte bis 1918 eine Doppelstellung als Hofbehörde einerseits und staatliche Behörde andererseits. Das wird schon an den Rechtsgrundlagen dieses Amtes deutlich. ${ }^{66}$ Hier standen staatliche (die Hofdekrete von 1785, 1795 und 1815, die JN 1852 und 1895, die kaiserliche VO 1916) und hausrechtliche (die

\footnotetext{
${ }^{66}$ Die Existenz des Obersthofmarschallamts wurde dabei vorausgesetzt und auch nicht in Frage gestellt. Die einzelnen Rechtsvorschriften beschäftigten sich daher nur mit dem Wirkungsbereich des Amtes.
}

einschlägigen Bestimmungen des Familienstatuts 1839) Regelungen nebeneinander, wobei nie eindeutig geklärt wurde, in welchem Verhältnis diese Vorschriften zueinander standen.

Auch an der rechtlichen Stellung des Personals wurde diese besondere Doppelstellung des Obersthofmarschallamts deutlich. Einerseits gab es die ständigen Bediensteten (den Kanzleidirektor, seinen Stellvertreter und die Konzeptsbeamten), die auch dienstrechtlich zum Hofstaat gehörten.67 Sie waren als Hofbedienstete weder Staatsbeamte noch Privatangestellte des Kaisers, vielmehr war der Dienst bei Hof ein öffentlicher Dienst sui generis, der organisatorisch vom sonstigen Staatsdienst völlig getrennt war. ${ }^{68}$ Andererseits waren an den Entscheidungen des Obersthofmarschallamts in streitigen Zivilsachen auch die von den Wiener Gerichtshöfen beigezogenen Richter beteiligt, und die waren Staatsbeamte. Für letztere galten die allgemeinen Voraussetzungen und dienstrechtlichen Vorschriften für Richter. Auch die hauptamtlichen Bediensteten des Obersthofmarschallamts mussten dem Richterstand angehören. ${ }^{69}$ Somit konnten nur (ehemalige) Richter an ordentlichen Gerichten hauptamtliche juristische Bedienstete des Obersthofmarschallamts werden.

Trotz dieser personellen Verflechtung blieb das Obersthofmarschallamt bis zum Ende der Monarchie organisatorisch völlig von den ordentlichen Gerichten getrennt. Obwohl es ausschließlich als erstinstanzliches Gericht fungierte, war das Amt organisatorisch keinem höherinstanzlichen Gerichtshof unterstellt und auch sonst nicht in die ordentliche Justizverwaltung eingebunden. Diese organisatorische Trennung wurde auch in der kaiserlichen Verordnung von 1916 betont: In $\S 4$ wurde ausdrücklich festgehalten, dass das Obersthofmarschallamt mit

\footnotetext{
${ }^{67}$ STROBL, Obersthofmarschallamt 123-124.

68 ZOLGER, Hofstaat 277-290.

${ }^{69}$ STROBL, Obersthofmarschallamt 123-124; § 1

RGBl. 223/1916.
} 
seinen Rechtsmittelgerichten (Oberlandesgericht Wien und Oberster Gerichtshof) nicht auf dem normalen Dienstweg, sondern nur durch deren Präsidenten verkehren sollte.

Das Obersthofmarschallamt als letzter Rest der Patrimonialgerichtsbarkeit überlebte sogar das Ende der Monarchie - wenn auch nur um wenige Monate. Die Provisorische Nationalversammlung hatte zwar schon am Tag der Ausrufung der Republik, am 12. November 1918, „alle Gesetze und Gesetzesbestimmungen, durch die dem Kaiser und den Mitgliedern des kaiserlichen Hauses Vorrechte zugestanden werden" aufgehoben. ${ }^{70}$ Das umfasste wohl auch die Abschaffung des besonderen Gerichtsstands des Kaiserhauses vor dem Obersthofmarschallamt. Rechtsangelegenheiten des Kaisershauses waren zwar die wichtigste Aufgabe des Amtes, aber nicht die einzige. Wegen dieser Zuständigkeiten (Gerichtsstand vor dem Obersthofmarschallamt als Privileg, Exterritoriale) war es nötig, ein eigenes Gesetz zu beschließen, mit dem die Kompetenzen des Obersthofmarschallamts an die ordentlichen Gericht übertragen wurde. ${ }^{71}$ Das geschah durch ein kurzes Gesetz vom 5. Februar 1919, das mit demselben Tag in Kraft trat.72 Damit war die Geschichte des Obersthofmarschallamts - abgesehen von reinen Abwicklungsmaßnahmen - beendet.

70 StGBl. 5/1918, Art. 5.

${ }^{71} \mathrm{RV}$, StenProt ProvNV, 1. Session, Blg. 144,.

72 StGBl. 87/1919.

\section{Korrespondenz:}

DDr. Christoph Schmetterer

Universität Wien

Institut für Rechts- und Verfassungsgeschichte

Abteilung KRGÖ

Strohgasse $45 / 2 d$

1030 Wien

christoph.schmetterer@univie.ac.at

\section{Abkürzungen:}

$\begin{array}{ll}\text { AußStrG } & \text { Außerstreitgesetz } \\ \text { EGJN } & \text { Einführungsgesetz zur JN } \\ \text { EO } & \text { Exekutionsrdnung } \\ \text { JN } & \text { Jurisdiktionsnorm }\end{array}$

Siehe auch das allgemeine Abkürzungsverzeichnis: [http://www.rechtsgeschichte.at/files/abk.pdf]

\section{Literatur:}

Wladimir AICHELBURG, Erzherzog Franz Ferdinand von Österreich-Este 1863-1914. Notizen zu einem ungewöhnlichen Tagebuch eines außergewöhnlichen Lebens, 3 Bde. (Horn-Wien 2014).

Adolf BACHRACH, Die Regierungsvorlage betreffend ein Gesetz über die Ausübung der Gerichtsbarkeit durch das Obersthofmarschallamt, in: JBl. 40 (1911) 38-40.

Edmund BERNATZIK, Die österreichischen Verfassungsgesetze mit Erläuterungen (Wien 21911).

Franz HAUKE, Kaiserliches und königliches Haus, in: Ernst MisCHLER, Josef UlBRICH (Hgg.), Österreichisches Staatswörterbuch, Bd. 3 (Wien 1907) 6-7.

Michael LOSCHELDER, Die österreichische allgemeine Gerichtsordnung von 1781. Grundlagen und Kodifikationsgeschichte (=Schriften zur Rechtsgeschichte 18, Berlin 1978).

Werner OGRIS, Die Rechtsentwicklung in Cisleithanien 1848-1918, in: Adam WANDRUSZKA, Peter URBANITSCH (Hgg.), Die Habsburgermonarchie 1848-1918, Bd. 2: Verwaltung und Rechtswesen (Wien 1975).

Ilse Reiter-ZatlouKal, Die Kontroverse um die Staatsbürgerschaft der HabsburgerInnen in der Ersten Republik, in: BRGÖ 6 (2016) 99-128.

Christoph SCHMETTERER, Die Rechtsstellung der Mitglieder des österreichischen Kaiserhauses von 1839 bis 1918, in: Journal on European History of Law 2 (2011/1) 15-20. 
Christoph SCHMETTERER, Die letztwilligen Verfügungen Kaiser Franz Josephs, in: BRGÖ 1 (2011) 317338.

Ferdinand SCHMID, Das Heeresrecht der österreichisch-ungarischen Monarchie (Wien 1903).

Emil SCHRUTKA Edler von Rechtenstamm, Die Regierungsvorlage betreffend ein Gesetz über die Ausübung der Gerichtsbarkeit durch das Obersthofmarschallamt, in: JBl. 40 (1911) 37-38.

Eduard STROBL Ritter von Albeg, Obersthofmarschallgericht in: Ernst MISCHLER, Josef ULBRICH (Hgg.), Österreichisches Staatswörterbuch, Bd. 3 (Wien 1907) 731-735.
Eduard STROBL Ritter von Albeg, Das Obersthofmarschallamt Sr. k.u.k. Apostol. Majestät (= Forschungen zur inneren Geschichte Österreichs 4, Innsbruck 1908).

Alfred WALDSTÄtTEN, Staatliche Gerichte in Wien seit Maria Theresia. Beiträge $\mathrm{zu}$ ihrer Geschichte (= Forschungen und Beiträge zur Wiener Stadtgeschichte 54, Innsbruck-Wien 2011).

Martina WinKelHOFER, Der Alltag des Kaisers. Franz Joseph und sein Hof (Innsbruck 2001).

Ivan Ritter von ZoLGER, Der Hofstaat des Hauses Österreich (= Wiener Staatswissenschaftliche Studien 14, Wien-Leipzig 1917). 